\title{
A Rare Case of Signet Cell Carcinoma of Transverse Colon in a Young Patient With Ulcerative Colitis
}

\author{
Mujtaba Mohamed ${ }^{\mathrm{a}, \mathrm{c}}$, Alsadiq Al Hillan ${ }^{\mathrm{a}}$, Eugene Zurkovsky ${ }^{\mathrm{a}}$, Min Zheng ${ }^{\mathrm{a}}$, \\ Arif Asif ${ }^{a}$, Reza Akhtar ${ }^{\mathrm{a}}$, Mohammad Hossain ${ }^{\mathrm{a}}$, Nikolaos T. Pyrsopoulos ${ }^{\mathrm{b}}$
}

\begin{abstract}
Signet ring cell carcinoma (SRCC) represents an extremely rare histological type for colon cancer, accounting for less than $1 \%$ of all colon neoplasms. SRCC is usually aggressive and it is associated with poor prognosis. It can be divided into mucinous adenocarcinoma (MAC) with signet cells and signet cell of more than $50 \%$ of the tumor area. Main differential diagnosis is metastatic gastric signet cell carcinoma. A 27-year-old African American man with past medical history of ulcerative colitis on mesalamine presented to emergency department with complaints of diarrhea abdominal pain and shortness of breath for 3 weeks. Physical exam was remarkable for tachycardia with a heart rate (HR) of 106 and pallor otherwise normal. Laboratory data showed potassium $2.8 \mathrm{mmol} / \mathrm{L}$, creatinine $1.11 \mathrm{mg} / \mathrm{dL}$, lactic acid 8 $\mathrm{mmol} / \mathrm{dL}$, hemoglobin $2 \mathrm{~g} / \mathrm{dL}$ and white blood cell count $21 \times 10^{6} / \mu \mathrm{L}$. Computed tomography (CT) scan of the abdomen with intravenous (IV) contrast showed distention of the colon, air-fluid levels and loss of haustra. Clostridium difficile stool PCR was positive. A clinical diagnosis of toxic megacolon due to $C$. difficile infection was made. Packed red blood cells (PRBCs) were transfused, and oral vancomycin and IV fluids were started. Symptoms and labs initially improved. However, on the third day, abdominal pain recurred with lactic acidosis. Diagnosis of refractory ulcerative colitis was made. Surgery was performed. Subtotal colectomy and ileostomy were done. Pathology revealed stage IV invasive signet ring cell adenocarcinoma, in the transverse colon poorly differentiated, with background of marked ulcerative colitis. Patient was started on adjuvant chemotherapy oxaliplatin, leucovorin and 5-flurouracil as an outpatient. Patient is undergoing 12 rounds of chemotherapy; he is currently in round 8 without complications. Patient is scheduled for screening colonoscopy and reversal of colostomy after completion of chemotherapy. SRCC of the colorectum is very rare, comprising less than $1 \%$ of colorectal cancer cases. It occurs mainly on the right colon and presents at later stages. Despite the rarity of this tumor, it is associated with ulcerative colitis.
\end{abstract}

Manuscript submitted April 13, 2020, accepted April 24, 2020

${ }^{a}$ Department of Medicine, Jersey Shore University Medical Center, Hackensack Meridian Health, Neptune, NJ 07753, USA

bDepartment of Gastroenterology and Hepatology, Rutgers New Jersey Medical School, Newark, NJ, USA

${ }^{c}$ Corresponding Author: Mujtaba Mohamed, Internal Medicine Residency Program, Jersey Shore University Medical Center, Hackensack Meridian Health, Neptune, NJ 07753, USA. Email: mujtaba.mohamed@hackensackmeridian.org

doi: https://doi.org/10.14740/jmc3469
The main differential diagnosis is a metastasis from gastric signet cell carcinoma. It is associated with a poor prognosis.

Keywords: Signet ring cell carcinoma; Colorectal cancer; Ulcerative colitis

\section{Introduction}

Signet ring cell carcinoma (SRCC) was reported for the first time in 1951 by Laufman and Saphir [1]. Since then, only a few hundred cases have been reported. It was reported mostly in Asian patients as sporadic case reports and small case series [2]. Colorectal SRCC is very rare, comprising less than $1 \%$ of colorectal cancer (CRC) cases [3]. SRCC and mucinous adenocarcinoma (MAC) with signet cells are two overlapping entities. SRCC can occur within the pools of MAC or in a diffusely infiltrative process with minimal extracellular mucin in a linitis plastica [4]. On the other hand, the World Health Organization (WHO) defined MAC as a carcinoma confirmed by $>50 \%$ of extracellular mucin pools that contain malignant epithelial or individual tumor cells including SRCC [4]. SRCC mainly occurs on the right side of the colon [5]. The main differential diagnosis is metastatic gastric carcinoma with SRCC, and it is necessary to rule out this diagnosis by endoscopy before finalizing a diagnosis of primary colorectal SRCC. SRCC is associated with poor prognosis. Despite the rarity of SRCC, it is more frequently associated with patients with ulcerative colitis [6]. Herein, we present a rare case of metastatic signet cell carcinoma of the colon in a young patient with ulcerative colitis.

\section{Case Report}

The patient was a 27-year-old African American man with past medical history of ulcerative colitis diagnosed 2 years prior to this presentation, on mesalamine. Patient presented to the emergency department with the complaints of generalized body aches, weakness, chest discomfort for 3 weeks, and palpitations with mild exertional dyspnea for 3 days. He had few episodes of bloody diarrhea 2 days prior to this presentation. He did not have jaundice, fever, nausea or vomiting, hematemesis or h/o recent weight loss. Vital signs showed blood 

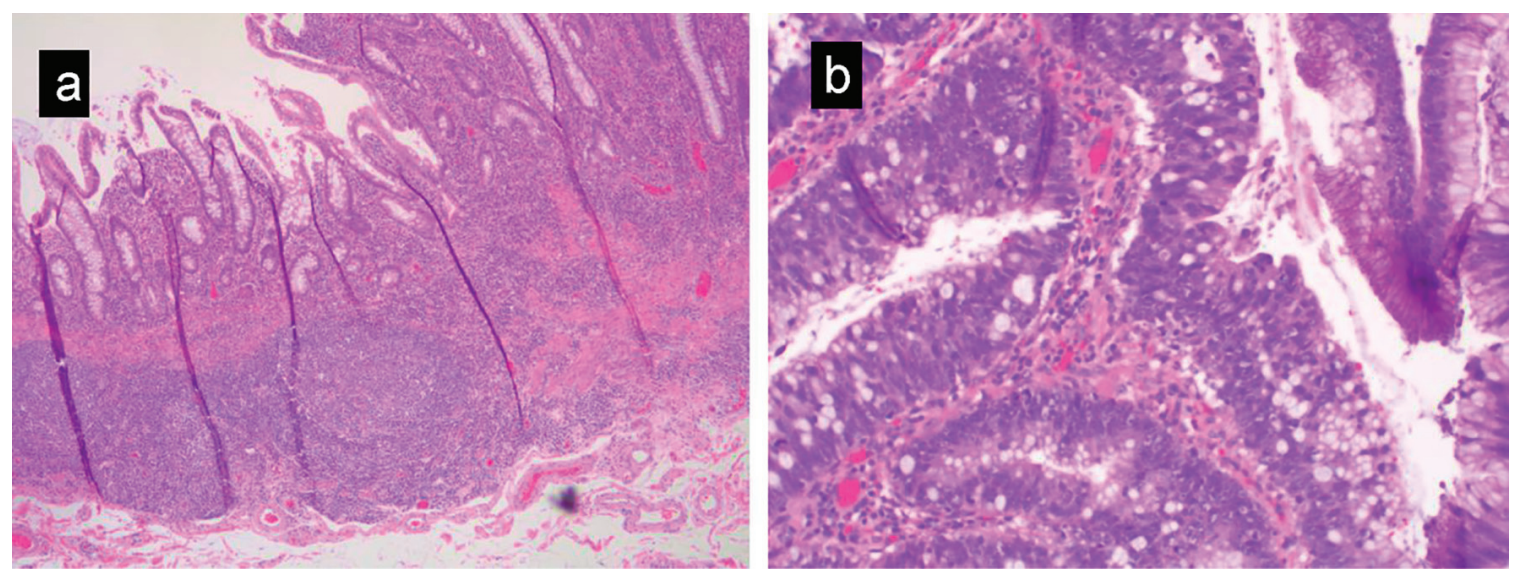

Figure 1. (a) Low magnification view showing inflammatory change in ulcerative colitis, including crypt distortion, acute and chronic inflammation, and ulceration. (b) High-grade glandular dysplasia characterized by nuclear hyperchromasia, loss of nuclear polarity and increased mitotic activity.

pressure (BP) of 90/62 $\mathrm{mm} \mathrm{Hg}$, heart rate of 109 beats $/ \mathrm{min}$, sinus tach, temperature of $36.4{ }^{\circ} \mathrm{C}$, respiratory rate (RR) of 16 breaths per minute and pulse oximetry of $99 \%$ on room air. Other physical examination was unremarkable except for generalized and conjunctival pallor. Initial laboratory investigation revealed serum potassium $2.8(3.5-5.2 \mathrm{mmol} / \mathrm{L})$, serum creatinine $1.11(0.44-1.0 \mathrm{mg} / \mathrm{dL})$, alkaline phosphatase 640 (38 - $126 \mathrm{IU} / \mathrm{L})$, elevated lactic acid $8(0.5$ - $2.0 \mathrm{mmol} / \mathrm{dL})$, severe anemia hemoglobin of $2(12-17.5)$, aspartate transaminase (AST) 87 (10 - 42 IU/L) and alanine transaminase (ALT) 85 (10 - $60 \mathrm{IU} / \mathrm{L}$ ). Computed tomography (CT) scan of the abdomen with intravenous (IV) contrast showed distention of the colon containing air-fluid levels and loss of haustra. Clostridium difficile PCR test was positive. Diagnosis of toxic megacolon was due to $C$. difficile infection and CT scan findings. The patient was admitted to the intensive care unit (ICU) for close monitoring. He received a total of four units of packed red blood cells (PRBCs). He was started on oral vancomycin and IV fluids. On the following day, the patient symptomatically improved with resolution of leukocytosis and lactic acidosis. Liver ultrasound was normal. On the fourth day of admission, the patient complained of abdominal pain and fever. Repeat labs showed leukocytosis and elevated lactate 3.5 reference value $(0.5$ - 2.0 $\mathrm{mmol} / \mathrm{dL}$ ). A diagnosis of refractory ulcerative colitis failing medical treatment was made. The patient underwent subtotal colectomy and ileostomy with Hartmann pouch and a small bowel resection. Surgical pathology of the subtotal colectomy showed severe ulcerative colitis. There was a multifocal highgrade glandular dysplasia arising from the inflammatory background in the transverse colon (Fig. 1a, b). A 15-mm focus of invasive signet ring cell adenocarcinoma, poorly differentiated in the transverse colon stage invading subserosa and involving two of 45 pericolic lymph nodes arising from a background of ulcerative colitis was reported (Fig. 2a-d). Metastatic signet cell carcinoma of the stomach was ruled out based on a normal endoscopy that the patient had 2 months prior to this presentation as a workup for iron deficiency anemia. The patient had a port inserted and was started on adjuvant chemotherapy oxaliplatin, leucovorin and 5-flurouracil as an outpatient by his oncologist.
Patient is undergoing 12 rounds of chemotherapy. He is currently in round 8 without complications. Patient is scheduled for screening colonoscopy and reversal of colostomy upon completion of chemotherapy.

\section{Discussion}

CRC is the third most common cancer. In the USA, annually, approximately 145,600 new cases of large bowel cancer are diagnosed, of which 101,420 are colon and the remainder are rectal cancers [7]. Annually, approximately 50,630 Americans die of CRC, accounting for approximately $8 \%$ of all cancer deaths [6]. More than $90 \%$ of CRC cases are adenocarcinomas [8]; however, several histological variants have been distinguished such as mucinous, signet ring cell, medullary, adenosquamous, micropapillary, spindle cell and undifferentiated [8]. Signet cell carcinoma is a subtype of colon cancer. The name SRCC is a descriptive term describing a carcinoma cell retaining abundant intracytoplasmic mucin that causes the nucleus to be pushed to the periphery of the cell. In affected sites, the tumor infiltrates the entire wall, therefore results in a rigid and contracted structure called the linitis plastica [5]. The majority of these tumors arise in the stomach. However, it was also described in breast, lung, bladder, pancreas, gallbladder and the colon. It is a rare tumor comprising less than $1 \%$ of all CRC cases [2]. SRCC is predominant in males with a ratio of 2:1. Belli et al reported the most common location is the right side of the colon [9]. Our patient is a young man and had the tumor on the transverse colon. These tumors can be classified into either MAC with signet cell (a subtype of MAC) or SRCC. WHO definition of SRCC is carcinoma confirmed by $>50 \%$ of SRCC but states that SRCC can occur within the pools of MAC or in a diffusely infiltrative process with minimal extracellular mucin in a linitis plastica pattern [9]. Luis et al described in his retrospective study that $68.7 \%$ of SRCC cases occur in the right colon, $9.4 \%$ in the transverse and $21.9 \%$ in the left colon [5]. Our patient had the tumor on the transverse colon. Of the patients, $43.7 \%$ present with metastasis, whereas 

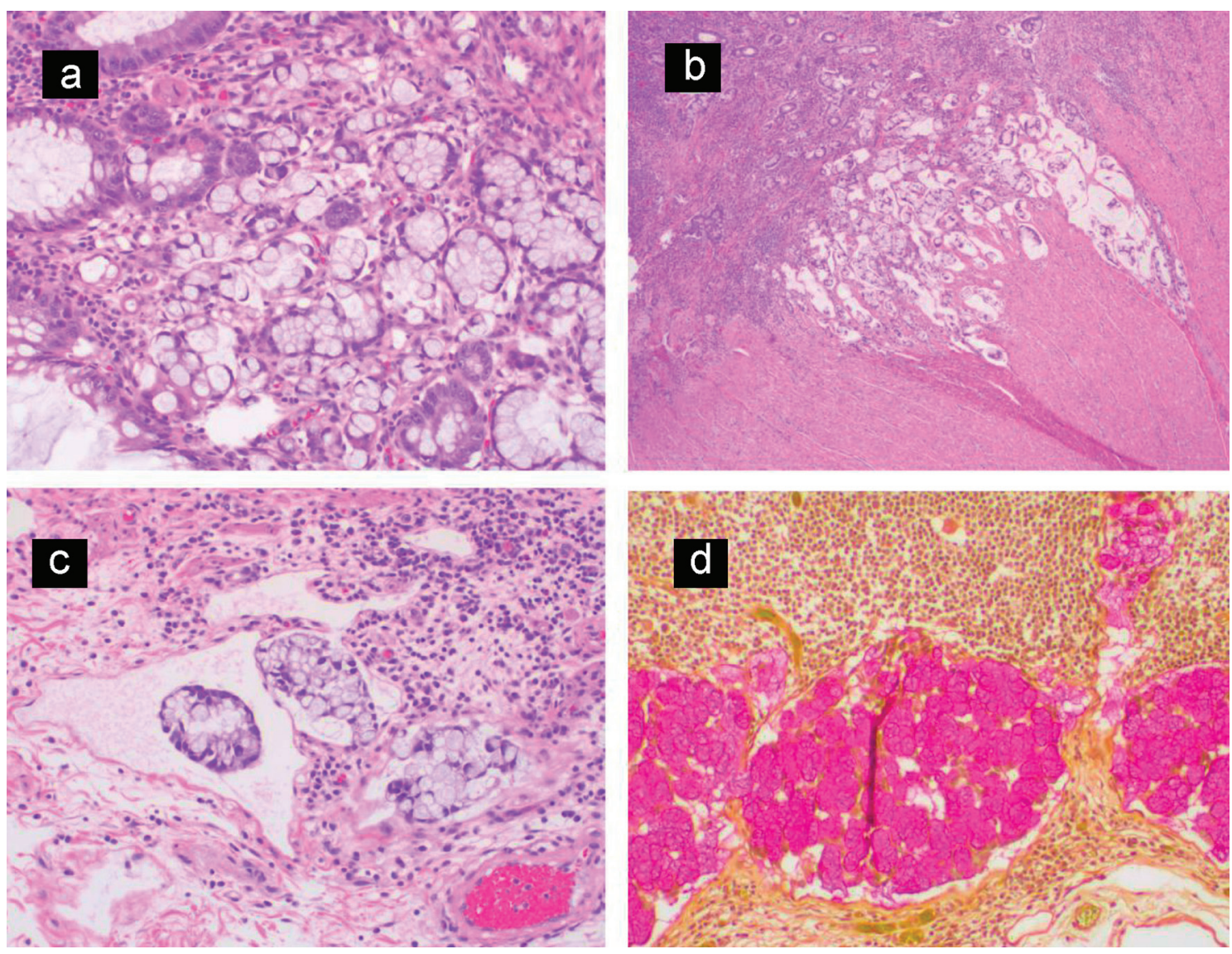

Figure 2. (a) Invasive nests of signet ring cell carcinoma arising from colonic mucosa. (b) Low magnification view showing signet ring cell carcinoma invading the muscularis propria of the colonic wall. (c) Lymphatic tumor invasion. (d) Mucicarmine stain highlighting cytoplasmic mucin of the metastatic signet ring cell carcinoma in a pericolic lymph node.

$64.3 \%$ of cases metastasize to the peritoneum, $46.5 \%$ present with lymph node metastasis (LNM) (mean of 14.3 positive lymph nodes), $62.5 \%$ with lymphovascular invasion and $25 \%$ with perineural invasion [5]. However, in the same study when SRCC was compared to MAC with signet cell, SRCC showed some distinctive characteristics. Patients presented a decade later ( 65 versus 56.5 years in comparison to MAC) as well as, in a higher clinical stage [5].

The pathogenesis of colorectal SRCC has not been fully understood yet. Tendon et al reported adenoma to carcinoma sequence for colorectal SRCC [10]. However, it is believed that SRCC may have a different mode of pathogenesis [11]. Hartman et al reported that precursor adenomatous lesions were more frequently observed in mucin-rich SRCC, and not in mucin-poor SRCCs [12]. There is an association between ulcerative colitis and SRCC. Colitis-associated carcinogenesis (CAC) leads to development of cancer through a different carcinogenic pathway known as the inflammation-metaplasia-dysplasia-carcinoma sequence with earlier identified molecular changes in TP531 [6].

Genetically, microsatellite instability (MSI) and loss of heterozygosity are present in SRCCs, similar to Lynch-associated tumors. Together they represent about $30 \%$ of tumors with these genetic features [12]. The impact of MSI on survival in colorectal SRCC has not been confirmed to have a prognostic value on survival [12]. It is most likely due to small size of previous studies. Further research is required to confirm or reject this hypothesis [13]. In our patient DNA mismatch repair (MMR) protein analysis was performed. Analyses for MLH-1, MSH-2, MSH-6 and PMS2 were all normal. Mutations in the ras gene family (K-ras, H-ras and N-ras) are found in about $50 \%$ of CRCs. Ninety-five percent of ras mutations are identified at k-ras gene, with $80 \%$ at codon 12 and $15 \%$ at codon 13 [13]. There are limited data in the literature on ras gene mutations in colorectal SRCC.

Colitis-associated signet cell carcinoma in situ is associated with alterations of TP53 and $C D H 1$ genes, resulting in a low expression of E-cadherin protein which impairs cell-cell adherence junctions and cell-proliferation signaling pathways. E-cadherin promoter methylation, the downregulation of Ecadherin expression, and aneuploidy have been frequently observed in ulcerative colitis-associated dysplasia [14].

Our patient's SRCC cells showed positive staining for CK20 and CDX2, and negative staining for CK7. MUC2 is negative and MUC5AC is positive in $41.7 \%$ of the cases. In our patient, intracytoplasmic mucin was positive for mucicarmine stain. MACs of the predominant signet ring cell type had the worst overall survival [15]. A high proportion of extracellular mucin and signet ring cell morphology in the non-muci- 
nous component is also associated with poor overall survival [15]. Other negative prognostic factors include age older than 65 years, lymphovascular invasion (LVI), perineural invasion (PNI), advanced stage and an absence of MSI.

The main differential diagnosis is a metastatic gastric carcinoma with SRCC, and it is necessary to rule out this diagnosis before finalizing a diagnosis of primary colorectal SRCC. It is difficult to differentiate between the two tumors based on immunohistochemical staining because one half to two-thirds of gastric SRCCs express MUC2, MUC5AC, CK20 and CK7; 90\% are CDX2-positive [16]. Our patient had an endoscopy done beforehand to assess microcytic anemia and it was negative for any pathology. Studies have shown that SRCCs of the colorectum respond poorly to current cytotoxic treatments and have lower rates of curative resection and poorer outcome so that new approaches are needed [17]. However, our patient is receiving 12 cycles of adjuvant chemotherapy oxaliplatin, leucovorin and 5-fluorouracil with complete remission. Surgical management has a key role in the treatment of localized tumors. The impact of curative surgery on survival in metastatic CRC is well established [18]. However, this option is limited to liver and lung metastasis and there are no data regarding the influence of the histology on the outcome after surgery in this setting. Furthermore, SRCC patients more often develop peritoneal and ovarian metastases, which are less relevant for curative surgery. Niek Hugen et al reported that stage III patients with colon SRCC who were treated with adjuvant chemotherapy had better survival than patients who did not receive adjuvant chemotherapy with 5-year relative survival of 52\% versus $30 \%$ [19]. However, in spite of the results of this study, the evidence base for adjuvant chemotherapy is unclear because of the lack of randomized controlled studies.

\section{Conclusion}

SRCC of the colorectum is very rare, comprising between $<$ $1 \%$ and $2.4 \%$ cases of CRCs. It occurs mainly on the right colon and present on later stages. It is associated with ulcerative colitis. Sometime presentation of this rare tumor might get complicated by other infection and associated comorbidities. So, clinicians should consider this diagnosis in appropriate clinical scenario. Due to the rarity of the disease, no conclusive guidelines exist for the treatment of this type of tumors.

\section{Acknowledgments}

The authors would like to thank Chunwei, Ma (Liberian at Jersey Shore University Medical Center for the help providing us with other case reports of signet ring cell carcinoma to compare it to our case.

\section{Financial Disclosure}

None to declare.

\section{Conflict of Interest}

The authors declare that there is no conflict of interest regarding the publication of this paper.

\section{Informed Consent}

Written informed consent was obtained from the patient for publication of this case report and any accompanying images.

\section{Author Contributions}

Each author has individually been involved and participated in drafting the manuscript and revising it critically for important intellectual content and has given final approval of the version to be published. Each has agreed to be accountable for all aspects of the work in ensuring that questions related to the accuracy or integrity of any part of the work are appropriately investigated and resolved. $\mathrm{MH}, \mathrm{EZ}, \mathrm{RA}, \mathrm{MZ}, \mathrm{AA}$ and NP encouraged MM and AA to learn about signet ring carcinoma of colon, how it presents and its management. All authors discussed the medical literature. MM presented the idea, AA and MM wrote the manuscript with input from all authors.

\section{Data Availability}

The authors declare that data supporting the findings of this study are available within the article.

\section{References}

1. Laufman H, Saphir O. Primary linitis plastic type of carcinoma of the colon. Arch Surg. 1951;62:79-79.

2. Chen JS, Hsieh PS, Chiang JM, Yeh CY, Tsai WS, Tang $\mathrm{R}$, Changchien $\mathrm{CR}$, et al. Clinical outcome of signet ring cell carcinoma and mucinous adenocarcinoma of the colon. Chang Gung Med J. 2010;33(1):51-57.

3. Sultan I, Rodriguez-Galindo C, El-Taani H, Pastore G, Casanova M, Gallino G, Ferrari A. Distinct features of colorectal cancer in children and adolescents: a population-based study of 159 cases. Cancer. 2010;116(3):758765.

4. Hamilton SR, Bosman FT, Boffetta P, et al. Carcinoma of the colon and rectum. In: Bosman FT, Carneiro F, Hruban R, Theise ND (eds). WHO classification of Tumours of the digestive system. IARC, Lyon, 2009. p. 134-146.

5. Pozos-Ochoa LI, Lino-Silva LS, Leon-Takahashi AM, Salcedo-Hernandez RA. Prognosis of Signet Ring Cell Carcinoma of the Colon and Rectum and their Distinction of Mucinous Adenocarcinoma with Signet Ring Cells. A Comparative Study. Pathol Oncol Res. 2018;24(3):609616.

6. Sugimoto S, Naganuma M, Iwao Y, Matsuoka K, Shimo- 
da M, Mikami S, Mizuno S, et al. Endoscopic morphologic features of ulcerative colitis-associated dysplasia classified according to the SCENIC consensus statement. Gastrointest Endosc. 2017;85(3):639-646 e632.

7. Siegel RL, Miller KD, Jemal A. Cancer statistics, 2019. CA Cancer J Clin. 2019;69(1):7-34.

8. Kang H, O'Connell JB, Maggard MA, Sack J, Ko CY. A 10 -year outcomes evaluation of mucinous and signet-ring cell carcinoma of the colon and rectum. Dis Colon Rectum. 2005;48(6):1161-1168.

9. Belli S, Aytac HO, Karagulle E, Yabanoglu H, Kayaselcuk F, Yildirim S. Outcomes of surgical treatment of primary signet ring cell carcinoma of the colon and rectum: 22 cases reviewed with literature. Int Surg. 2014;99(6):691698.

10. Tandon M, Sostek M, Klein MA. Focus of signet ring cell carcinoma in an adenoma of the sigmoid colon. Arch Pathol Lab Med. 1999;123(10):957-959.

11. Ooi BS, Ho YH, Eu KW, Seow Choen F. Primary colorectal signet-ring cell carcinoma in Singapore. ANZ J Surg. 2001;71(12):703-706.

12. Hartman DJ, Nikiforova MN, Chang DT, Chu E, Bahary N, Brand RE, Zureikat AH, et al. Signet ring cell colorectal carcinoma: a distinct subset of mucin-poor microsatellite-stable signet ring cell carcinoma associated with dismal prognosis. Am J Surg Pathol. 2013;37(7):969-977.

13. Andreyev HJ, Norman AR, Cunningham D, Oates JR, Clarke PA. Kirsten ras mutations in patients with colo- rectal cancer: the multicenter "RASCAL" study. J Natl Cancer Inst. 1998;90(9):675-684.

14. Azarschab P, Porschen R, Gregor M, Blin N, Holzmann $\mathrm{K}$. Epigenetic control of the E-cadherin gene (CDH1) by $\mathrm{CpG}$ methylation in colectomy samples of patients with ulcerative colitis. Genes Chromosomes Cancer. 2002;35(2):121-126.

15. Song IH, Hong SM, Yu E, Yoon YS, Park IJ, Lim SB, $\mathrm{Kim} \mathrm{JC}$, et al. Signet ring cell component predicts aggressive behaviour in colorectal mucinous adenocarcinoma. Pathology. 2019;51(4):384-391.

16. Chu PG, Weiss LM. Immunohistochemical characterization of signet-ring cell carcinomas of the stomach, breast, and colon. Am J Clin Pathol. 2004;121(6):884-892.

17. Lee WS, Chun HK, Lee WY, Yun SH, Cho YB, Yun HR, Park SH, et al. Treatment outcomes in patients with signet ring cell carcinoma of the colorectum. Am J Surg. 2007;194(3):294-298.

18. Hugen N, Verhoeven RH, Lemmens VE, van Aart CJ, Elferink MA, Radema SA, Nagtegaal ID, et al. Colorectal signet-ring cell carcinoma: benefit from adjuvant chemotherapy but a poor prognostic factor. Int J Cancer. 2015;136(2):333-339.

19. Van Cutsem E, Cervantes A, Nordlinger B, Arnold D, Esmo Guidelines Working Group. Metastatic colorectal cancer: ESMO Clinical Practice Guidelines for diagnosis, treatment and follow-up. Ann Oncol 2014;25(Suppl. 3):1-9. 